OPEN ACCESS

Edited by:

Baokai Cui,

Beijing Forestry University, China

Reviewed by:

Shaobin Zhong,

North Dakota State University, United States

Zonghua Wang,

Fujian Agriculture and Forestry

University, China

*Correspondence:

Baoshan Chen

chenbs2008@163.com

Zide Jiang

zdjiang@scau.edu.cn

${ }^{\dagger}$ Co-first authors

Specialty section:

This article was submitted to

Fungi and Their Interactions,

a section of the journal

Frontiers in Microbiology

Received: 28 June 2019

Accepted: 28 August 2019

Published: 10 September 2019

Citation:

Zhu G, Deng Y, Cai E, Yan M, Cui G, Wang Z, Zou C, Zhang B, Xi P, Chang C, Chen B and Jiang Z (2019) Identification and Functional Analysis of the Pheromone Response Factor

Gene of Sporisorium scitamineum.

Front. Microbiol. 10:2115.

doi: 10.3389/fmicb.2019.02115

\section{Identification and Functional Analysis of the Pheromone Response Factor Gene of Sporisorium scitamineum}

\author{
Guining Zhu ${ }^{1,2 t}$, Yizhen Deng ${ }^{1 t}$, Enping Cai ${ }^{1}$, Meixin Yan ${ }^{1,2}$, Guobing Cui ${ }^{1}$, \\ Zhiqiang Wang ${ }^{3}$, Chengwu Zou ${ }^{3}$, Bin Zhang ${ }^{1}$, Pinggen Xi', Changqing Chang ${ }^{1}$, \\ Baoshan Chen $^{3 *}$ and Zide Jiang ${ }^{1 *}$
}

${ }^{1}$ Guangdong Province Key Laboratory of Microbial Signals and Disease Control, State Key Laboratory for Conservation and Utilization of Subtropical Agro-Bioresources, Department of Plant Pathology, South China Agricultural University, Guangzhou, China, ${ }^{2}$ Guangxi Key Laboratory of Biology for Crop Diseases and Insect Pests, Plant Protection Research Institute, Guangxi Academy of Agricultural Sciences, Nanning, China, ${ }^{3}$ State Key Laboratory for Conservation and Utilization of Subtropical Agro-Bioresources, College of Life Science and Technology, Guangxi University, Nanning, China

The sugarcane smut fungus Sporisorium scitamineum is bipolar and produces sporidia of two different mating types. During infection, haploid cells of opposite mating types can fuse to form dikaryotic hyphae that can colonize plant tissue. Mating and filamentation are therefore essential for S. scitamineum pathogenesis. In this study, we obtained one T-DNA insertion mutant disrupted in the gene encoding the pheromone response factor (Prf1), hereinafter named SsPRF1, of S. scitamineum, via Agrobacterium tumefaciensmediated transformation (ATMT) mutagenesis. Targeted deletion of SsPRF1 resulted in mutants with phenotypes similar to the T-DNA insertion mutant, including failure to mate with a compatible wild-type partner strain and being non-pathogenic on its host sugarcane. qRT-PCR analyses showed that SSPRF1 was essential for the transcription of pheromone-responsive mating type genes of the a1 locus. These results show that SsPRF1 is involved in mating and pathogenicity and plays a key role in pheromone signaling and filamentous growth in S. scitamineum.

Keywords: Sporisorium scitamineum, pheromone response factor, mating, pathogenicity, fungi

\section{INTRODUCTION}

Sugarcane smut caused by Sporisorium scitamineum is a devastating disease in sugarcane worldwide. Plants infected with the pathogen are severely stunted with thin stalks, producing no millable canes. The most recognizable characteristic of this disease is a black or gray growth from the top of plant cane that is referred to as a "smut whip" that is composed of a central core of pithy plant tissue surrounded by the fruiting structures of the fungus and the brown to black teliospores (Croft and Braithwaite, 2006; Sundar et al., 2012). S. scitamineum is bipolar and produces sporidia of two opposite mating types, MAT-1 and MAT-2 (Yan et al., 2016b). Sporidia of different mating types can fuse to form pathogenic dikaryotic hyphae to infect sugarcane buds and the hyphae grow within the meristematic tissue, eventually producing whip-like fruiting structure and teliospores in the infected plants. The diploid teliospores germinate and undergo meiosis to yield haploid sporidia, which need to mate again to infect the plant and to initiate a next round of infection (Albert and Schenck, 1996; Croft and Braithwaite, 2006; Yan et al., 2016b). Thus, mating plays a central role in the life cycle of smut pathogens, as it initiates parasitism by a morphological and 
physiological transition from saprotrophic yeast cells to pathogenic filaments (Hartmann et al., 1996; Bakkeren et al., 2008; Kellner et al., 2011).

The conserved MAPK and CAMP/PKA signaling pathways regulate important aspects of fungal virulence in various pathogenic fungi such as Magnaporthe oryzae (MarroquinGuzman and Wilson, 2015), U. maydis (Mayorga and Gold, 1999), and Setosphaeria turcica (Li et al., 2016) etc. The life cycle of S. scitamineum is similar to that of the well-studied model fungus Ustilago maydis that causes corn smut disease. In $U$. maydis, mating is regulated by two loci, $a$ and $b$. The biallelic $a$ locus (a1 and a2) encodes pheromone precursors $m f a 1$ and $m f a 2$, respectively, and receptors pra1 and pra2, respectively. The pheromone-receptor system is required for recognition and fusion of haploid sporidia, while the multiallelic $b$ locus encodes $b E$ and $b W$, subunits of a heterodimeric transcription factor that regulates filamentation, dikaryon maintenance and pathogenicity. The expression of the genes in the $a$ and $b$ loci is induced upon pheromone stimulation (Hartmann et al., 1996). Basal as well as pheromone-induced transcription of these mating type genes is regulated by the pheromone responsive factor Prf1, which binds specifically to the pheromone response elements (PREs) located in the vicinity of all pheromone-inducible genes at the $a$ and $b$ loci (Hartmann et al., 1996; Urban et al., 1996). The activity of Prf1 is, in turn, regulated by the cyclic-AMP (cAMP) signaling pathway and the mitogen-activated protein (MAP) kinase module (Regenfelder et al., 1997; Krüger et al., 1998; Müller et al., 1999, 2003; Andrews et al., 2000; Kaffarnik et al., 2003). Prf1 is also regulated by other regulators such as rop1, hap2 and med1 (Brefort et al., 2005; Zarnack et al., 2008; Mendoza-Mendoza et al., 2009; Chacko and Gold, 2012).

In recent years, the whole genome sequences of $S$. scitamineum strains from China, Brazil, Australia and South Africa have been reported (Que et al., 2014; Taniguti et al., 2015; Dutheil et al., 2016). The availability of genome sequences facilitates the investigation of the pathogenicity mechanism of S. scitamineum. To understand the molecular basis of sexual mating and pathogenic development in S. scitamineum, we developed an efficient Agrobacterium tumefaciens-mediated transformation (ATMT) system (Sun et al., 2014) and identified the $b$-locus as essential for sexual mating and filamentous growth in S. scitamineum (Yan et al., 2016b). During the screening of an ATMT transformant library, we found a T-DNA insertion mutant, 248E3, that was unable to mate or form filamentous hyphae. The disrupted gene was identified as an ortholog of the $U$. maydis PRF1 gene, designated as SsPRF1. Functional characterization of $S S P R F 1$ revealed that it functions as a pheromone response regulator, plays an essential role in the regulation of a locus gene expression, and is required for S. scitamineum pathogenicity.

\section{MATERIALS AND METHODS}

\section{Strains and Growth Conditions}

The compatible haploid strains JG35 (MAT-2) and JG36 (MAT1) of S. scitamineum were isolated from germinated teliospores collected from the sugarcane smut in Guangxi, China (Lu et al., 2017). Cultures were grown in YePS broth medium (1.0\% yeast extract, $2.0 \%$ peptone, $2.0 \%$ sucrose) on a rotary shaker at $200 \mathrm{rpm}$ at $28^{\circ} \mathrm{C}$ or on solid YePS agar at $28^{\circ} \mathrm{C}$.

\section{Plant Materials}

Sugarcane seedlings of the highly susceptible variety, ROC22, were cultivated in B. Chen's experimental field in Guangxi University, and used for pathogenicity assay.

\section{Molecular Manipulations}

Plasmid DNA was isolated with the SanPrep plasmid mini kit (Sangon, B518191) and S. scitamineum genomic DNA was extracted using the method described previously (Yan et al., 2016b). For Southern blot analysis, DNA samples (3-5 $\mu \mathrm{g})$ digested with appropriate restriction enzymes were separated by electrophoresis and blotted to Hybond $\mathrm{N}^{+}$membrane. Standard hybridization and detection protocols were performed using the method of DIG DNA labeling and detection kit (Roche, 11093657910). Total RNA was isolated with TRNzol-A ${ }^{+}$ (Tiangen, DP421) and first-strand cDNA was synthesized using the Revert AidFirst Strand cDNA Synthesis Kit (Thermo Fisher Scientific, K1621).

\section{ATMT Mutagenesis and Identification of T-DNA Insertion Site}

Agrobacterium tumefaciens-mediated transformation (ATMT) was used to generate mutants of $S$. scitamineum haploid strains JG35 and JG36 using the A. tumefaciens strain AGL1 (Sun et al., 2014). The transformation procedure was adapted and modified from the methods developed for $U$. maydis (Ji et al., 2010). T-DNA left-border flanking sequence was amplified by high-efficiency TAIL-PCR (hiTAIL-PCR; Bölker et al., 1992; Liu and Chen, 2007), using genomic DNA of T-DNA insertion mutants as templates. Two rounds of amplifications were performed. Specific primers (LB1, LB2, and LB3) in combination with arbitrary degenerate primer (LAD1-4) and AC1 were used. The primer pair LB1/LAD1-4 was used in the pre-amplification step, while the primer pairs AC1/LB2 and $\mathrm{AC} 1 / \mathrm{LB} 3$ were used in the primary and secondary-amplification, respectively. Primer sequences are listed in Table 1. The primary TAIL PCR product of about $850 \mathrm{bp}$ was purified using the PCR-Clean kit (Sangon, SK8142), cloned into pMD 18$\mathrm{T}$ Vector (TaKaRa, 6011), and sequenced using M13 forward or reverse primer.

\section{Plasmid Construction for SsPRF1 Deletion and Complementation}

Binary vectors pEX1-GAP-eGFP, pNGR1, and pEX2, all derived from the binary T-DNA vector pPZP200 (Hajdukiewicz et al., 1994) and with T-DNA, were kindly provided by Dr. Lianghui Ji from National University of Singapore (Ji et al., 2010). SsPRF1 deletion construct was made according to protocol previously described (Ji et al., 2010). Briefly, a 1577 bp upstream fragment (HS1) and a 841 bp downstream fragment (HS2) of the SsPRF1 gene were amplified with primer pairs prf1-P1/prf1-P2 and 
TABLE 1 | Primers used in this study.

\begin{tabular}{|c|c|}
\hline Name & Sequence \\
\hline LAD1-4 & $\begin{array}{l}\text { 5'ACGATGGACTCCAGAGCGGCCGC(G/C/T)(G/A/T)N(G/C/T) } \\
\text { NNNCGGT }\end{array}$ \\
\hline $\mathrm{AC} 1$ & 5'ACGATGGACTCCAGAG \\
\hline LB1 & 5'TGACGGCAATTTCGATGATGCAGC \\
\hline LB2 & 5'GGACCGATGGCTGTGTAGAAGTAC \\
\hline LB3 & 5'CGATCGACAAGCTCGAGTTTCTCC \\
\hline M13-R & 5'TCACACAGGAAACAGCTATGACC \\
\hline M13-F & 5'CAGGGTITTCCCAGTCACGAC \\
\hline $\mathrm{hpH} 1-\mathrm{F}$ & 5'GCAAGACCTGCCTGAAACCG \\
\hline $\mathrm{hpH} 1-\mathrm{R}$ & 5'GGTCAAGACCAATGCGGAGC \\
\hline prf1-P1 & 5'AAAGTTAAAACTGCTCTGTGCCACGCCTTGA \\
\hline prf1-P2 & 5'AAACTGCAGCTGACTACAGACGATGTTGGTGGT \\
\hline prf1-P3 & 5'AAAACTAGTCTGTAGGATGCAATGTATAGGC \\
\hline prf1-P4 & 5'AAAGGATCCTGGAAGGTTGGTGCGAGA \\
\hline prf1-P5 & 5'CACTAGTGGTACAACACCGAC \\
\hline prf1-P6 & 5'GCAAACCTGCCTATCAGCAAG \\
\hline prf1-P7 & 5'GTGTGAGTAGTTCCCAGATAAGGG \\
\hline prf1-P8 & 5'AGGTGTGAAAACGATGCGATG \\
\hline 248E3-F & 5'ATGCGAGACCAAGCTACCAC \\
\hline 248E3-TR & 5'CGTAATGGGCACGATCTTCGG \\
\hline prf1-2R & 5'AAAGGATCCCTACGTGTAGTGTCCATTCCAA \\
\hline Nat-F & 5'CACTCTTGACGACACGGCTT \\
\hline Nat-R & 5'GCATGCTCATGTAGAGCGC \\
\hline 248E3-qF & 5'GTCGACCTCTITCACGGATG \\
\hline 248E3-qR & 5'CTCGCTTGGGAAAGGAGATG \\
\hline pra1-qF & 5'CGAGTGTTGCCATGTTGGAGAGT \\
\hline pra1-qR & 5'TTGTAGCCTTGACGAACTTCCTGAC \\
\hline mfa1-qF & 5'TCTITACCCAGACCGCCCAGAC \\
\hline mfa1-qR & 5'GGTGCAGCTAGAGTAGCCAAGG \\
\hline $\mathrm{bE} 1-\mathrm{qF}$ & 5'TGAAAGTTCTCATGCAAGCC \\
\hline$b E 1-q R$ & 5'TGAGAGGTCGATTGAGGTTG \\
\hline bW1-qF & 5'CACGTTGGATCTCGCTCGGT \\
\hline bW1-qR & 5'TCGGAAGGGAGGACGCAAAC \\
\hline $18 \mathrm{~S}-\mathrm{qF}$ & 5'GACACCTCAACTCAGCGACACAA \\
\hline $18 \mathrm{~S}-\mathrm{qR}$ & 5'TGCCCTTGCCATAGTCCCAAATG \\
\hline 248E3-F3 & 5'GAGAAGGCTACGAGCCAGTT \\
\hline 248E3-R & 5'GCAAATGTAGTGCAATGACGC \\
\hline mfa1-P1 & 5'AAAGTTTAAACGCTGTGTTGTTGATTGAGAGTGG \\
\hline mfa1-P2 & 5'AAAAAGCTTGTTGGTGTTCGCAAGAACGA \\
\hline mfa1-P3 & 5'AAAACTAGTTGCTGACGAATGTGTCCTTC \\
\hline mfa1-P4 & 5'AAAGGATCCCGATTGTGATAGTGTGAGAGAGAG \\
\hline mfa1-P5 & 5'TCAAGCGATAATGCAGCCAGTC \\
\hline mfa1-P8 & 5'GGATGGCTTCATCGTGTGTACTGT \\
\hline pra1-P1 & 5'AAAGTTTAAACAGGTAGACGCATCCGATCCA \\
\hline pra1-P2 & 5'AAACTGCAGGACAAGCCGAAGTGGTGATG \\
\hline pra1-P3 & 5'AAAACTAGTACATGGCTTCGTTGTCGAGT \\
\hline pra1-P4 & 5'AAAGGATCCAGCTGTGCTCTTCTTGCTCTT \\
\hline pra1-P5 & 5'GAGAACCTTGAGCAAGTGCTCG \\
\hline pra1-P8 & 5'CGAGAAATGGTGTCACAAGACGAT \\
\hline pra1-F & 5'ATGCTTGACCACGTAACACCT \\
\hline pra1-R & 5'CGCAGATATCGAGGTAGTCACA \\
\hline
\end{tabular}

prf1-P3/prf1-P4, respectively (Table 1) using JG36 genomic DNA as template. The HS2 fragment was digested with SpeI and BamHI and ligated into pEX2 to produce pEX2-HS2. The HS1 fragment was digested with PmeI and PstI and then ligated into pEX2-HS2 at the corresponding sites to yield the gene knock out construct pEX2- $\triangle \operatorname{Prf1}$. The recombinant plasmid pEX2- $\triangle$ Prf1 was introduced into Agrobacterium strain AGL1 and used for transformation of JG35 and JG36 sporidia (Sun et al., 2014). Transformants were screened by PCR using primer pairs prf1-P5/prf1-P6, prf1-P7/prf1-P8, and 248E3-F/248E3-TR (Table 1), respectively. Locations of the primers were illustrated in Figure 3B. Gene knockout and absence of ectopic copy were further confirmed by Southern blot analysis as described.

For functional complementation assays, the primer pairs prf1-P1/prf1-2R (Table 1) were used to amplify a $4627 \mathrm{bp}$ fragment carrying the entire SsPRF1 gene from JG36 genomic DNA. The PCR product was digested with PmeI and BamHI and cloned into the Nourseothricin-resistance vector pNGR1 at the corresponding sites to yield the complementary plasmid pCPrf1. The plasmid pCPrf1 was introduced into Agrobacterium strain AGL1 and used for transformation of the T-DNA insertion mutant $248 \mathrm{E} 3$.

\section{Mating Assays}

Assay for mating was performed as previously described (Yan et al., 2016b). The S. scitamineum transformants were picked from the selection plates and inoculated in YePS medium (supplemented with $300 \mu \mathrm{g} / \mathrm{ml}$ Cefotaxime) for 2 days at $28^{\circ} \mathrm{C}$ on a rotary shaker. The 2 days cultures of wild-type opposite mating type strains (JG35, JG36, or mutants) were mixed with equal amounts of cells and a small drop was spotted on YePS-agar plate and incubated at $28^{\circ} \mathrm{C}$ for observation of colony morphology.

\section{Pathogenicity Assay}

Pathogenicity assay was performed using the method described by Yan et al. (2016b). Fungal strains or mutants were grown in YePS broth for 2 days in a shaking incubator at $28^{\circ} \mathrm{C}$ and then collected by centrifugation to remove the medium. Fungal cells were resuspended in sterilized double-distilled water adjusted to $1 \times 10^{6}$ cells $/ \mathrm{ml}$ before mixing with the opposite mating type for inoculation. Sugarcane seedlings of the highly susceptible variety, ROC22, at 5-6 leaf stage grown in greenhouse were inoculated by injecting the stem near the growing point with approximately $200 \mu \mathrm{l}$ of the mixed culture per plant. The control plants were injected with sterilized double-distilled water. Three biological repeats were applied in the inoculation and each repeat contained 5 plants grown in a pot of $30 \mathrm{~cm}$ in diameter. Pathogenicity was examined and documented till 120 days post-inoculation when the characteristic symptoms of a "smut whip" could be fully observed on the sugarcane plants inoculated with the positive control of JG36/JG35.

For fungal biomass assessment in the infected sugarcane seedlings, the sugarcane stem tissue infected by S. scitamineum sporidia (compatible mating types mixing) was collected at 72 hpi. Quantification of relative fungal biomass in infected sugarcane stem tissue was performed using the fungal ACTIN gene as reference, and sugarcane glyceraldehyde dehydrogenase $(G A P D H)$ gene as reference for normalization, following the established protocol (Sun et al., 2019). 


\section{Transcriptional Profiling}

Strains or mutants pre-grown in YePS broth for 2 days at $28^{\circ} \mathrm{C}$ with shaking were diluted to $0.1 \mathrm{OD}_{600}$ with YePS. Equal amounts of the diluted cells of JG35 were mixed with those of JG36, JG36 $\Delta$ prf1, and $248 \mathrm{E} 3$, respectively and $50 \mathrm{ml}$ of the mixtures or the haploid strains were incubated for 2 days at $28^{\circ} \mathrm{C}$ on a rotary shaker. Cultures were harvested by centrifugation and total RNA was isolated from haploid strains or mixture of strains. Two $\mu \mathrm{g}$ of purified total RNA of each sample was used as a template for first-strand cDNA synthesis using the RevertAid ${ }^{\mathrm{TM}}$ First Strand cDNA Synthesis Kit (Thermo Fisher Scientific, K1621). For real-time quantitative polymerase chain reaction (qRT-PCR) assay, the cDNA samples were diluted 20-fold with $\mathrm{ddH}_{2} \mathrm{O}$ and used as qRT-PCR templates. The specific primer pairs of 248E3-qF/248E3-qR, mfa1-qF/mfal-qR, pra1-qF/pra1-qR, and $18 \mathrm{~S}-\mathrm{qF} / 18 \mathrm{~S}-\mathrm{qR}$ were used to amplify the target genes, SsPRF1, SsMFA1, SsPRA1, and $18 S r R N A$, respectively. $18 S$ rRNA was used as the reference gene for expression normalization of the target genes and wild-type strain JG36 was used as the calibrator. qRTPCR was performed in a LightCycler ${ }^{\circledR} 480$ Real-time PCR system (Roche). Amplification reaction contained $10 \mu \mathrm{l}$ of $2 \times$ SYBR Green I Master Mix (Roche, 4707516001), $1.0 \mu \mathrm{l}$ of each primer $(10 \mu \mathrm{M}), 1.0 \mu \mathrm{l}$ of template cDNA and nuclease-free water was added to a final volume of $20 \mu$ l. The cycling parameters were: $95^{\circ} \mathrm{C}$ for $10 \mathrm{~min}$, followed by 50 cycles of $95^{\circ} \mathrm{C}$ for $10 \mathrm{~s}$ and $60^{\circ} \mathrm{C}$ for $60 \mathrm{~s}$. Then, the PCR products were heated to $95^{\circ} \mathrm{C}$ with $4.4^{\circ} \mathrm{C} / \mathrm{s}$, cooled to $65^{\circ} \mathrm{C}$ with $2.2^{\circ} \mathrm{C} / \mathrm{s}$, and heated to $97^{\circ} \mathrm{C}$ with $0.11^{\circ} \mathrm{C} / \mathrm{s}$. Negative controls were reactions without template or transcriptase and were included in each experiment set. qRTPCR reactions were performed for three technical replicates and three biological replicates for each sample. For comparison of gene expression, the average threshold cycle $(\mathrm{Ct})$ values for target genes and the house-keeping gene 18s rRNA were first calculated and then relative quantification was calculated using the comparative $2^{-\Delta \Delta \mathrm{Ct}}$ method (Livak and Schmittgen, 2001). Data were normalized with endogenous 18s $r R N A$ level from the same samples.

\section{RESULTS}

\section{Characterization of the T-DNA Insertion Mutant 248E3}

To identify genes essential for $S$. scitamineum mating/filamentation, we constructed an ATMT mutant library with two compatible mating-type strains of S. scitamineum, JG36 (MAT-1), and JG35 (MAT-2). A total of 25056 transformants with hygromycin resistance marker were obtained, in which 15873 transformants were derived from JG36 and 9183 from JG35. All the T-DNA insertion mutants exhibited bright green fluorescence under epifluorescent microscope (Figure 1A), confirming the presence of a T-DNA cassette carrying an eGFP in the S. scitamineum genome (Supplementary Figure S1A).

We then screened these transformants for mating defects. Mixing of the wild-type strain JG36 (MAT-1) with the compatible wild-type mating partner JG35 (MAT-2) gave rise to a fluffy colony, producing visible thin and white aerial filaments at 12 days post-spotting on YePS-agar plate (Figure 1B), indicating successful mating and formation of dikaryotic hyphae. Six isolates showing glossy appearance of mating mixture, indicative of mating defects, were selected and subjected to a second round of mating test. Among these was the mutant 248E3 from JG36 background, which was unable to produce dikaryotic hyphae when co-spotted with JG35 (Figure 1B), confirming that it was defective in mating. Apart from mating defect, 248E3 appeared indistinguishable from the wild type strain JG36, in colony and basidiospore (sporidium) morphology (Figures 1B,C) and the growth rate in YePS liquid medium (Figure 1D; $p>0.05$ ).

\section{Identification of the S. scitamineum PRF1 Homolog}

Southern blotting analysis showed that mutant $248 \mathrm{E} 3$ contains a single copy of T-DNA inserted into its chromosome (Supplementary Figure S1B). By high-efficiency TAIL-PCR with the primers LAD1-4, AC1 paired with LB1, LB2 and LB3 (Table 1 and Supplementary Figure S1C), a fragment of about 850 bp was obtained using 248E3 genomic DNA as template (Supplementary Figure S1D). Sequence analysis showed that this fragment contained a $348 \mathrm{bp}$ stretch of the genome sequence. Using this $348 \mathrm{bp}$ fragment to screen the genome sequence of $S$. scitamineum in the NCBI database, we identified a putative gene (ID: SSCI14340.1), in which the T-DNA was inserted inside its coding sequence at coordinate $1033 \mathrm{bp}$ from the translation start codon (Supplementary Figure S1C). The open reading frame (ORF) of SSCI14340.1 was $2166 \mathrm{bp}$, encoding a predicted polypeptide of 722 amino acids (GenBank: CDW96669.1). This protein shows $78 \%$ identity to the probable pheromone response factor Prf1 of S. reilianum (GenBank: CBQ73103.1), 57\% to Prf1 of U. maydis (AAC32736.1) and $56 \%$ to a protein related to pheromone response factor Prf1 of $U$. hordei (CCF52951.1), respectively (Figure 2A). All these fungal Prf1 orthologs contain a highly conserved domain of HMG box superfamily at the $\mathrm{N}$-terminus (Figure 2B). Therefore we named this gene SsPRF1. By sequencing the RT-PCR products using total RNA from JG35 (MAT-2), we found that SsPRF1 was also present in the strain with the opposite mating type. Southern blotting analysis confirmed that this gene was present in both JG35 and JG36 with a single copy in their genomes (Supplementary Figure S1B).

\section{SsPRF1 Is Essential for Sexual Mating in S. scitamineum}

To further demonstrate the functionality of $S s P R F 1$, a $4.6 \mathrm{~kb}$ fragment containing the entire $S S P R F 1$ gene of $S$. scitamineum MAT-1 (JG36) strain was cloned into the vector pNGR1 to result in the complementation plasmid pCPrf1. This plasmid was introduced into the T-DNA insertion mutant $248 \mathrm{E} 3$ to generate the complementation transformant C248E3-34. Sporidia of the complementation strain C248E3-34 were able to form white fluffy colonies when co-spotted with a compatible wild-type mating partner JG35 (MAT-2), similar to the sexual mating between the two wild-type mating-type strains (Figure 3A). 
A

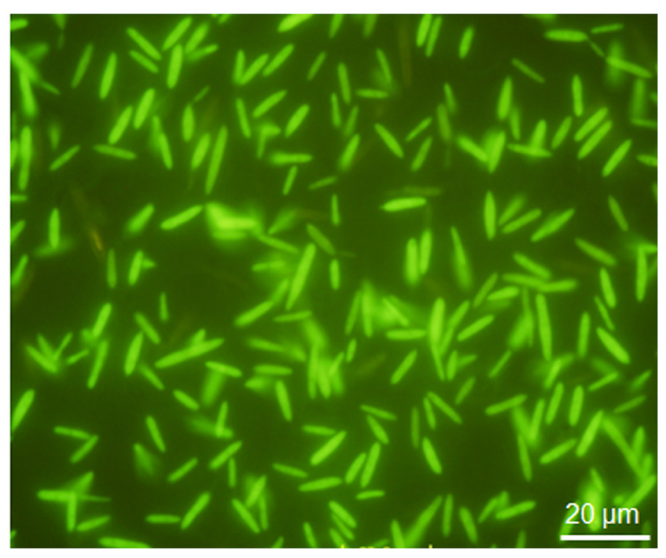

C

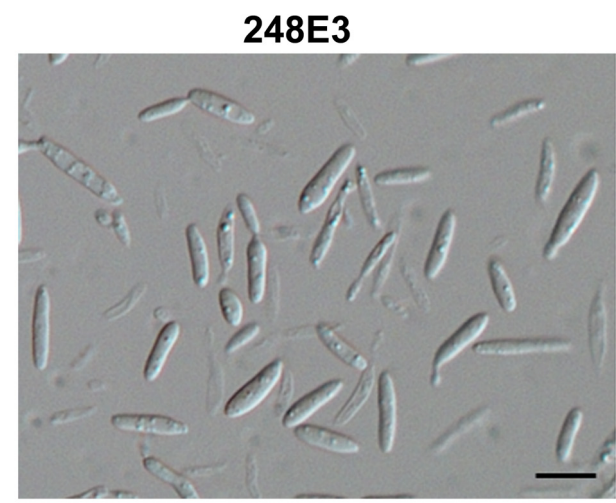

B

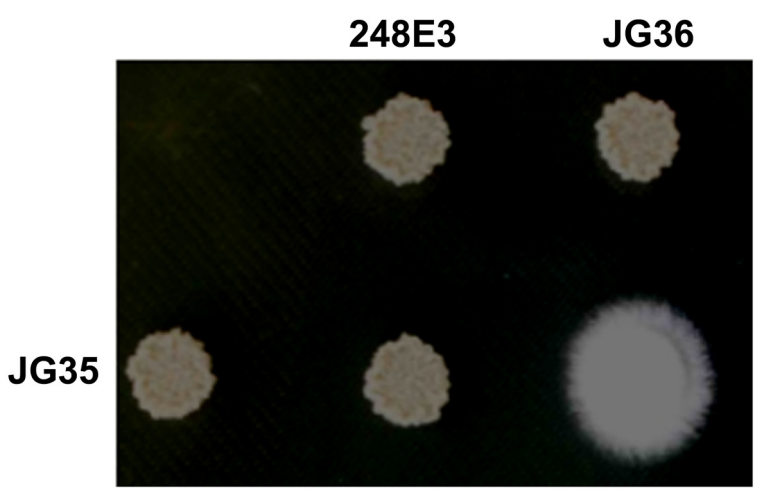

JG36

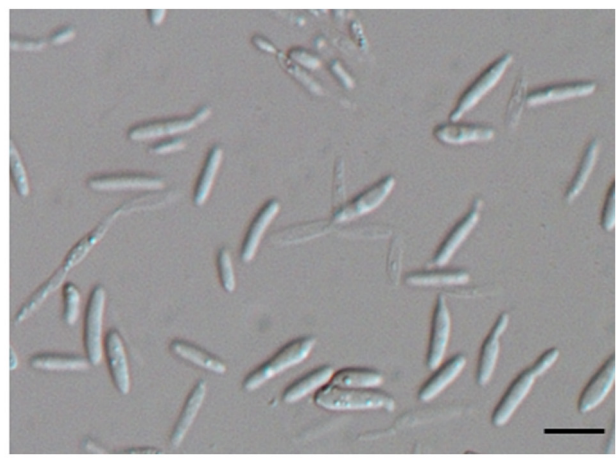

D

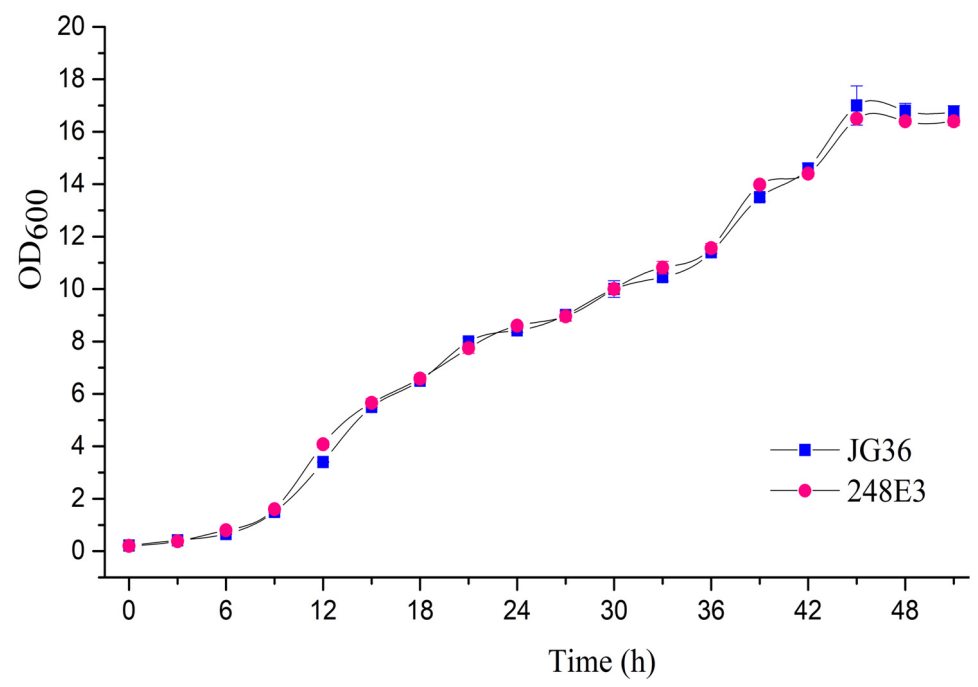

FIGURE 1 | Characterization of T-DNA insertion mutant 248E3. (A) Fluorescence imaging of the mutant haploid sporidia. Photographs were taken at day 3 after inoculation onto the YePS plates. Scale bar $=20 \mu \mathrm{m}$. (B) Mating behavior of mutant 248E3. The mutant was co-spotted with compatible partner JG35 on YePS plate and incubated at $28^{\circ} \mathrm{C}$ for 3 days. (C) Differential interference contrast (DIC) images of sporidia: mutant $248 \mathrm{E} 3$ and JG36 (wild type). Scale bar = $10 \mu \mathrm{m}$.

(D) Growth curves of mutant $248 \mathrm{E} 3$ and JG36 in YePS liquid medium. Cultures were kept at $28^{\circ} \mathrm{C}$ in a rotary incubator at 200 rpm. The initial $\mathrm{OD}_{600}$ value was 0.2 and was measured once every $3 \mathrm{~h}$. Data give averages \pm SE of three technical replicates conducted at the same time. 
A
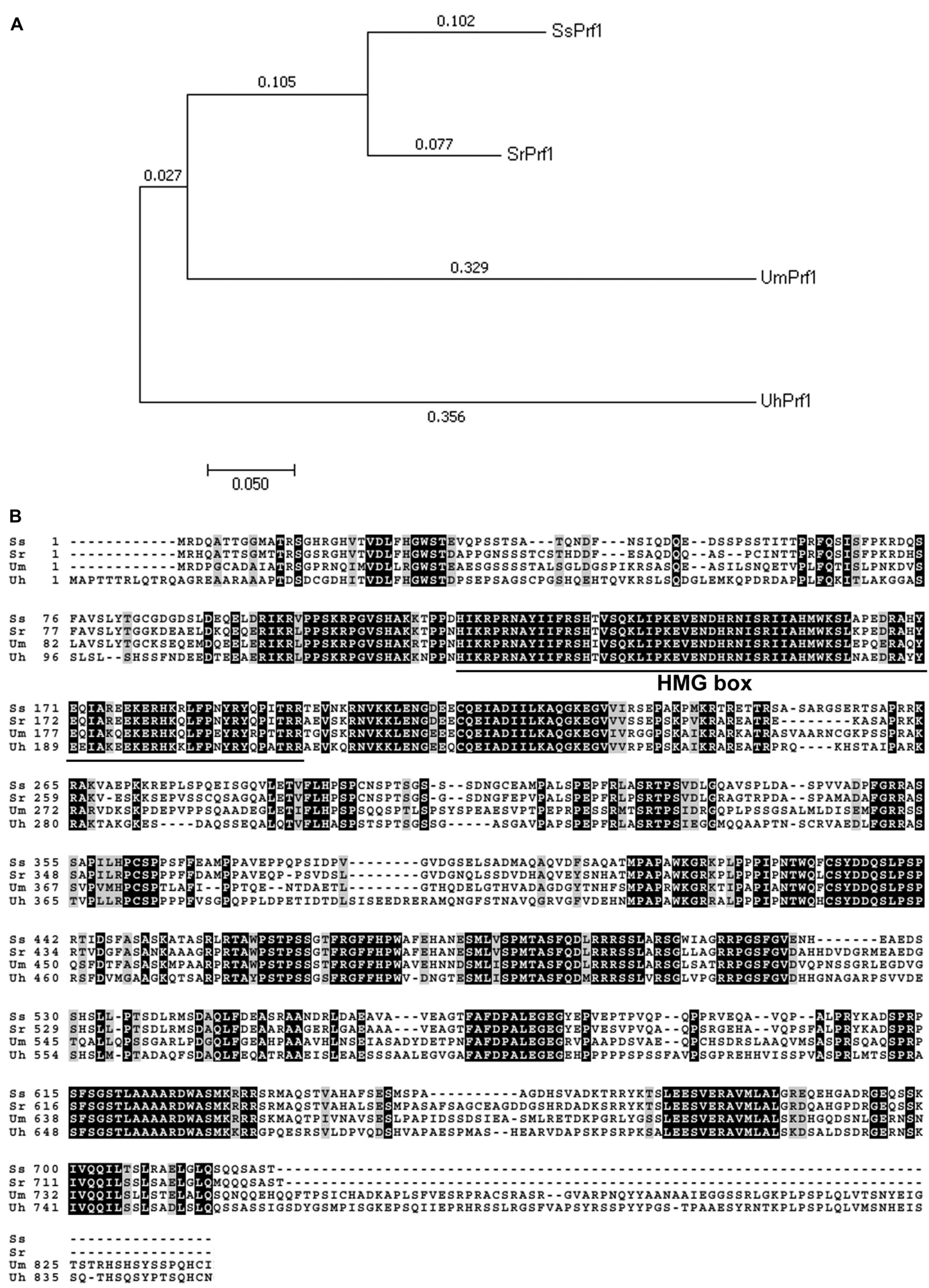

FIGURE 2 | Analysis of fungal PRF1 orthologs. (A) Phylogenetic analysis of four Prf1 orthologs in fungi was conducted in MEGA7 (Kumar et al., 2016) using the Neighbor-Joining method (Saitou and Nei, 1987). The optimal tree with the sum of branch length $=0.99597394$ is shown. The percentage of replicate trees in which the associated taxa clustered together in the bootstrap test (1000 replicates) is shown next to the branches (Felsenstein, 1985). The tree is drawn to scale, with branch lengths in the same units as those of the evolutionary distances used to infer the phylogenetic tree. The evolutionary distances were computed using the Jones-Taylor-Thornton (JTT) matrix-based method (Jones et al., 1992) and are in the units of the number of amino acid substitutions per site. SrPrf1: CBQ73103.1; UmPfr1: AAC32736.1; UhPrf1: CCF52951.1. (B) Sequence alignments of the above mentioned fungual SsPrf1 proteins was performed using Boxshade server (https://embnet.vital-it.ch/software/BOX_form.html), with the aligned sequence generated by Clustal Omega (www.ebi.ac.uk/Tools/services/web/toolresult.ebi? jobld=clustalo-I20180725-032536-0068-98009260-p2m\&showColors=true\&tool=clustalo). A conserved domain (MATA_HMG-box, class I member of the HMG-box superfamily of DNA-binding proteins) is underlined. 


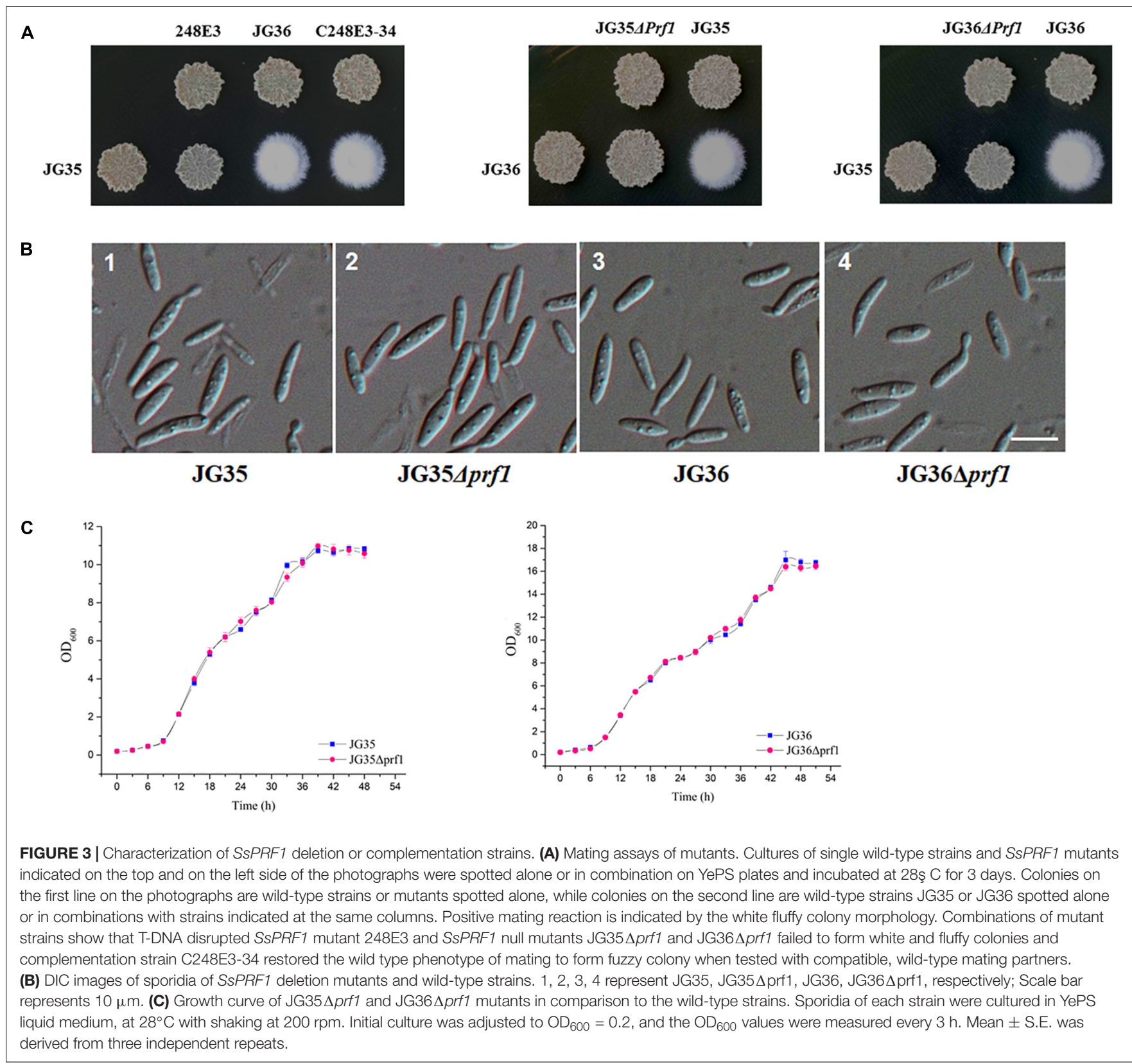

We further generated the SsPRF1 null mutants in both JG35 and JG36 background by homologous recombination (Supplementary Figure S2A). After antibiotic selection and two rounds of successive screening by mating tests, the SsPRF1 null mutants JG35 $\Delta$ prf1 from JG35 and JG36 $\Delta$ prf1 from JG36 were verified by PCR amplification (Supplementary Figures S2B,C) and confirmed by Southern blotting (Supplementary Figure S2D). In agreement with the phenotype of the T-DNA insertion mutant 248E3, the morphology of these SsPRF1 deletion mutants appeared indistinguishable from the wild-type strain (Figure 3B), except that they both failed to form fluffy colonies when co-spotted with compatible wild-type mating partners (Figure 3A). The growth rates of the ssprf1D mutants in YePS liquid medium were also comparable to that in their wild-type strains (Figure 3C; $p>0.05$ ).

\section{SsPRF1 Is Required for S. scitamineum Pathogenicity}

To determine if SsPRF1 is involved in pathogenicity, we inoculated the sugarcane seedlings of variety ROC22 (highly susceptible to $S$. scitamineum) with the mixed sporidial cells of $S$. scitamineum in combinations of 248E3(MAT1)/JG35(MAT-2), C248E3-34(MAT-1)/JG35(MAT-2), and JG36 $\Delta \operatorname{prf1}(M A T-1) / J G 35(M A T-2)$, with wild-type strains JG35(MAT-2)/JG36(MAT-1) as positive control. It was found 
that sugarcane seedlings inoculated with either the T-DNA disrupted SsPRF1 mutant (248E3) or the SsPRF1 null mutant (JG36 $\Delta p r f 1)$ remained healthy throughout the observed period of 120 days. In contrast, plants infected with wild-type combination JG36/JG35 displayed severe stunting, spindly stalks and upright and narrower leaves after 60 days, and whips emerged from the shoots at 90-120 days post-inoculation (Figure 4). In agreement with the restoration of mating, the complementation strain C248E3-34 incited the characteristic symptoms of "smut whip" when mixed with wild-type strain JG35 (MAT-2), comparable to those by the wild-type strains (Figure 4; $p>0.05$ ).

We further quantified the relative fungal biomass in infected sugarcane stem tissue, at 3 days post-infection (dpi), and our result showed that the relative fungal biomass in the sugarcane seedlings infected by JG36 $\Delta \operatorname{prf1}(M A T-1) / \mathrm{JG} 35$ (MAT2) was $139.65 \pm 72.12 \%$ of that in the ones infected by the wild-type JG35(MAT-2)/JG36(MAT-1) mixture. Similarly, the relative fungal biomass from the 248E3(MAT-1)/JG35(MAT2) infected seedlings was $118.21 \pm 24.21 \%$ of that of WT. Difference between WT infection and mutant infection was not significant $(p>0.05)$. This result indicates that colonization of the plant tissue by WT or mutant mixed spordia at early stage were comparable, however, we could not differentiate the in planta fungal biomass detected by this assay was in sporidial or hyphal form. This result at least rules out the possibility that failure of developing disease symptom in the mutant infected sugarcane seedlings was not due to insufficient inoculum at the beginning.

\section{SsPrf1 Regulates the Expression of Pheromone-Responsive Genes}

Recently, the whole genome sequences of $S$. scitamineum strains from China, Brazil, Australia and South Africa have been reported (Que et al., 2014; Taniguti et al., 2015; Dutheil et al., 2016). The presence of pheromone receptor gene SsPRA1 was first reported by Que et al. (2014) and the whole coding region was identified by RT-PCR and located to the a1 locus (Yan et al., 2016b). During our BLAST search against S. scitamineum genome, the pheromone precursor gene SsMFA1 with high identity to the pheromone precursor gene $m f a 1.2$ in $S$. reilianum was also identified and verified by RACE in the a1 locus of S. scitamineum (GenBank: CP010914.1, 857085-857204).

To further dissect the function of SsPRF1 in pheromone signaling and pathogenic development, transcription of the JG36specific genes SsMFA1 and SsPRA1, as well as the gene SsPRF1 that is present in both JG35 and JG36, were assessed in the haploid strains of JG35, JG36, JG36 $\Delta$ prf1, and 248E3, with or without pheromone induction from the opposite mating type strain. As shown in Figure 5A, SsPRF1 was detected at comparable levels in JG36 and JG35, while undetectable in JG36 $\Delta$ prf1, as expected. SsMFA1 and SsPRA1 expression was undetectable in JG35 (MAT-2) and was barely detectable in JG36 $\Delta$ prf1 or 248E3 (Figure 5A). However, it was interesting that the SsPRF1 transcript level was threefold up-regulated in 248E3, suggesting that (1) the truncated SsPRF1 was transcriptionally active, and (2) the mutated SsPRF1 showed enhanced mRNA transcription.

To investigate the induction effect of the opposite mating type strain on mating type-related gene expression, JG36, JG36 $\Delta$ prf1, and 248E3 were co-cultured with their compatible wild-type mating partner JG35 (MAT-2), which was assumed to provide $a 2$ pheromone and should stimulate the transcription of pheromone-inducible genes. As shown in Figure 5B, SsMFA1 expression was significantly elevated (10.7-fold) in JG35/JG36 combination but not in JG35/JG36 $\Delta$ prf1 or JG35/248E3 combinations. SsPRA 1 expression was up-regulated by 4.3 -fold in the JG35/JG36 combination but no increase in JG35/JG36 $\Delta$ prf1 or JG35/248E3 combinations could be detected, suggesting that SsPRF1 in JG35 could not complement the SsPRF1 defect in JG36 $\Delta$ prf1 and 248E3. It was interesting that SsPRF1 expression was stimulated in JG35/JG36 combination (2.4fold up-regulated) and in JG35/248E3 (1.9-fold up-regulated), implying that $S$ SPRF1 expression is regulated by signal(s) released during mating by the opposite mating type strains. In contrast, no stimulation in SsPRF1 transcription was seen in JG35/JG36 $\Delta p r f$, whose transcript level reached about $50 \%$ of the wild-type level for SsPRF1 mRNA. This reduced transcript level may likely be caused by the dilution of the SsPRF1-transcribing JG35 with SsPRF1-non-transcribing JG36 $\Delta p r f 1$.

We also assessed transcriptional regulation of the b locus genes $b E$ and $b W$ by qRT-PCR. Our result showed that transcription of $b E$ or $b W$ genes was slightly reduced in the sporidia of SsPRF1 disruption or deletion mutants (Figure 5A), but the difference was not significant $(p>0.05)$. Under pheromone-induction condition (JG35/JG36), the b locus genes were upregulated (bE1: 1.90-folds; bW1: 2.54-folds; $p<0.05$ ) while loss or disruption of SsPRF1 in JG36 background made it unable to induce b locus gene transcription when mixed with wild-type JG35 sporidia (Figure 5B; $p<0.05$ ). Overall, we conclude that SsPrf1 is responsible for transcriptional induction of $\mathrm{a}$ and $\mathrm{b}$ locus genes under mating condition.

\section{a Locus Genes Are Essential for S. scitamineum Mating/Filamentation}

To further confirm that S. scitamineum al locus genes are functional in mating/filamentation, we next deleted SsMFA1 and SsPRA1 respectively, in JG36 (MAT-1) background. PCR amplification of the gene of interest (SsMFA1 or SsPRA1) or the flanking sequences, using the primer pairs as indicated in Supplementary Figure S3A and listed in Table 1, confirmed successful gene deletion (Supplementary Figure S3B). We also performed Southern blot analysis to confirm deletion of SsPRA1 gene (Supplementary Figure S3C).

Sporidia of the wild-type JG36 (MAT-1), JG36 $m$ fa1, or JG36 $\Delta$ pra1 were respectively mixed and co-spotted with a compatible wild-type mating partner JG35 (MAT-2), to test their ability of mating/filamentation. We found that similar to the sexual mating between the $\triangle p r f 1$ mutant and wild-type matingtype strain, deletion of SsMFA1 or SsPRA1 led to failure of filamentation (Figure 6A). This confirms that S. scitamineum 


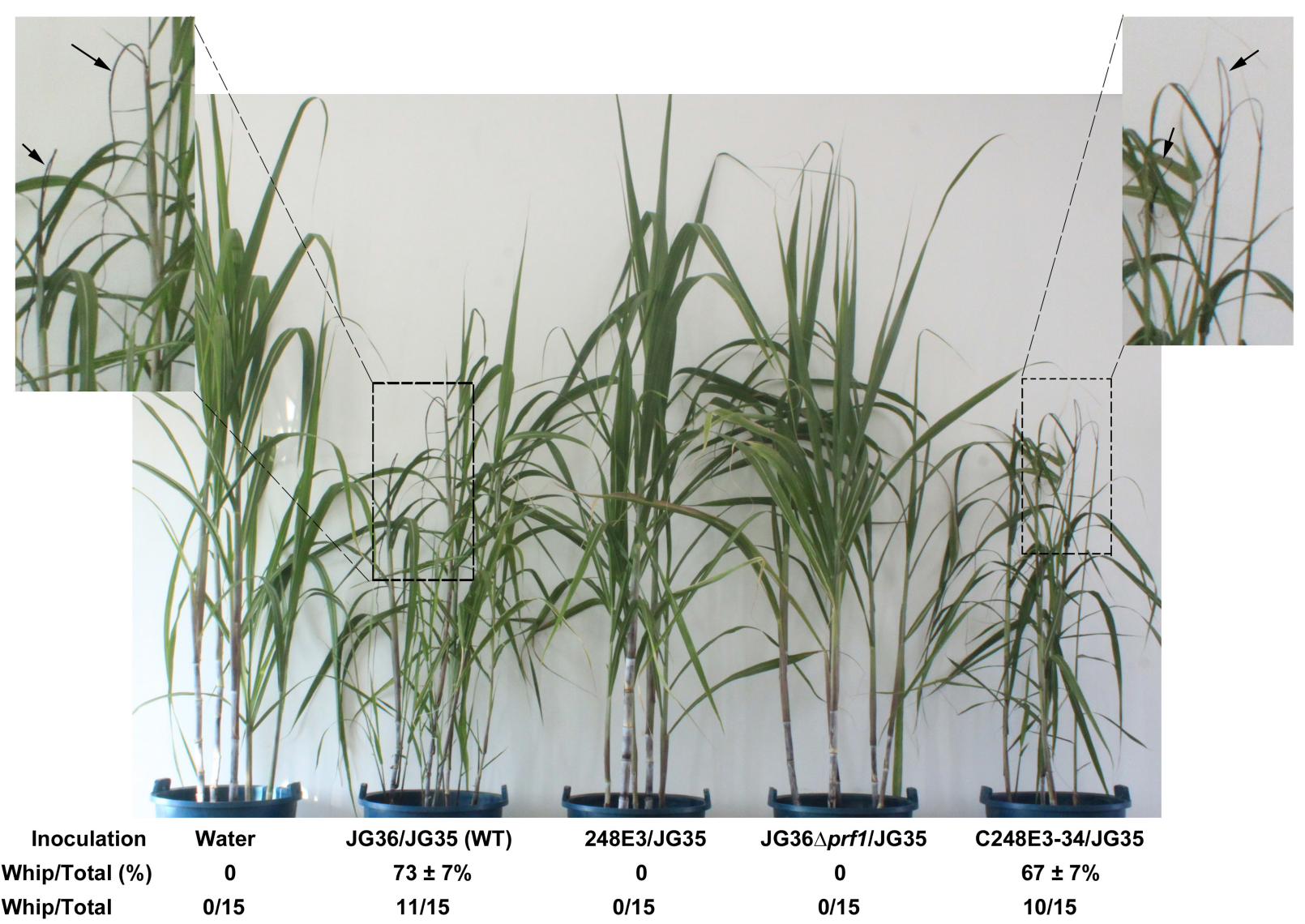

FIGURE 4 | Pathogenicity assay of SsPRF1 mutants. For pathogenicity assays, seedlings of the sugarcane variety ROC22 (highly susceptible to S. scitamineum) were inoculated by injecting the stem near the growing point with the mixed fungal cells of various pairwise combinations. Symptoms were documented and photographed at about 120 days post-inoculation. Dash-line boxed regions are enlarged for a better view of whip symptoms, denoted by arrows. Percentage of seedling showing whip formation out of total inoculated seedling was presented as Mean $\pm \mathrm{SE}$, derived from three independent repeats, each of which contained 5 seedlings. Number of whip/total seedlings was also indicated.

a locus gene SsMFA1 and SsPRA1 are functional in regulating fungal dimorphic switch, and likely acting at downstream of SsPrf1. This result is also consistent with a recent report on SsMFA1 characterization (Sun et al., 2019).

\section{SsPRF1 Is Independent/Downstream of cAMP-PKA Signaling Mediated ROS Regulation}

Chang et al. (2018) recently reported a cAMP-PKA signaling pathway regulating $S$. scitamineum intracellular redox homeostasis, to promote mating/filamentation and host infection. As in the corn smut fungus $U$. maydis, Prf1 was shown to act at downstream of both PKA and MAPK pathway and not involved in redox regulation (Kaffarnik et al., 2003), we here assessed the epistatic interaction between SsPrf1 and the cAMP-PKA pathway, and the role (if any) of SsPrf1 in redox regulation.

As the mating/filamentation defect of the cAMP-PKA mutants could be fully or partially restored by exogenous addition of cAMP (Chang et al., 2018), we first tested the effect of cAMP on $\Delta p r f 1$ mutant. The $\Delta p r f 1$ mutant remained un-mating or un-filamentous when mixed with the compatible wild-type JG35 (MAT-2) sporidia (Figure 6B). This indicates that SsPrf1 may act at downstream of cAMP-PKA signaling pathway, as consistent with what has been reported in U. maydis. Alternatively, SsPrf1 may act in parallel with cAMP-PKA signaling.

We also tested tolerance to the oxidative stress caused by $1 \mathrm{mM} \mathrm{H}_{2} \mathrm{O}_{2}$, in comparison between the wild-type and the $\Delta p r f 1$ sporidia, and found no obvious difference (Supplementary Figure S4A). We next measured the intracellular $\mathrm{H}_{2} \mathrm{O}_{2}$ of the $\Delta$ prf1 sporidia, and found that it was comparable to that of the wild-type sporidia (Supplementary Figure S4B; $p>0.05)$. Finally, exogenous addition of low concentration $(0.1 \mathrm{mM})$ of $\mathrm{H}_{2} \mathrm{O}_{2}$ could not restore the mating/filamentation of the $\Delta p r f 1$ mixed with the wild-type sporidia (Supplementary Figure S4C), as it does to the cAMP-PKA mutants (Chang et al., 2018). These results suggest that SsPrf1 may not be involved in redox regulation in S. scitamineum, which is at downstream of cAMP-PKA pathway. Therefore the role of SsPrf1 in S. scitamineum mating/filamentation is more likely due to transcriptional regulation of the mating-type genes. 


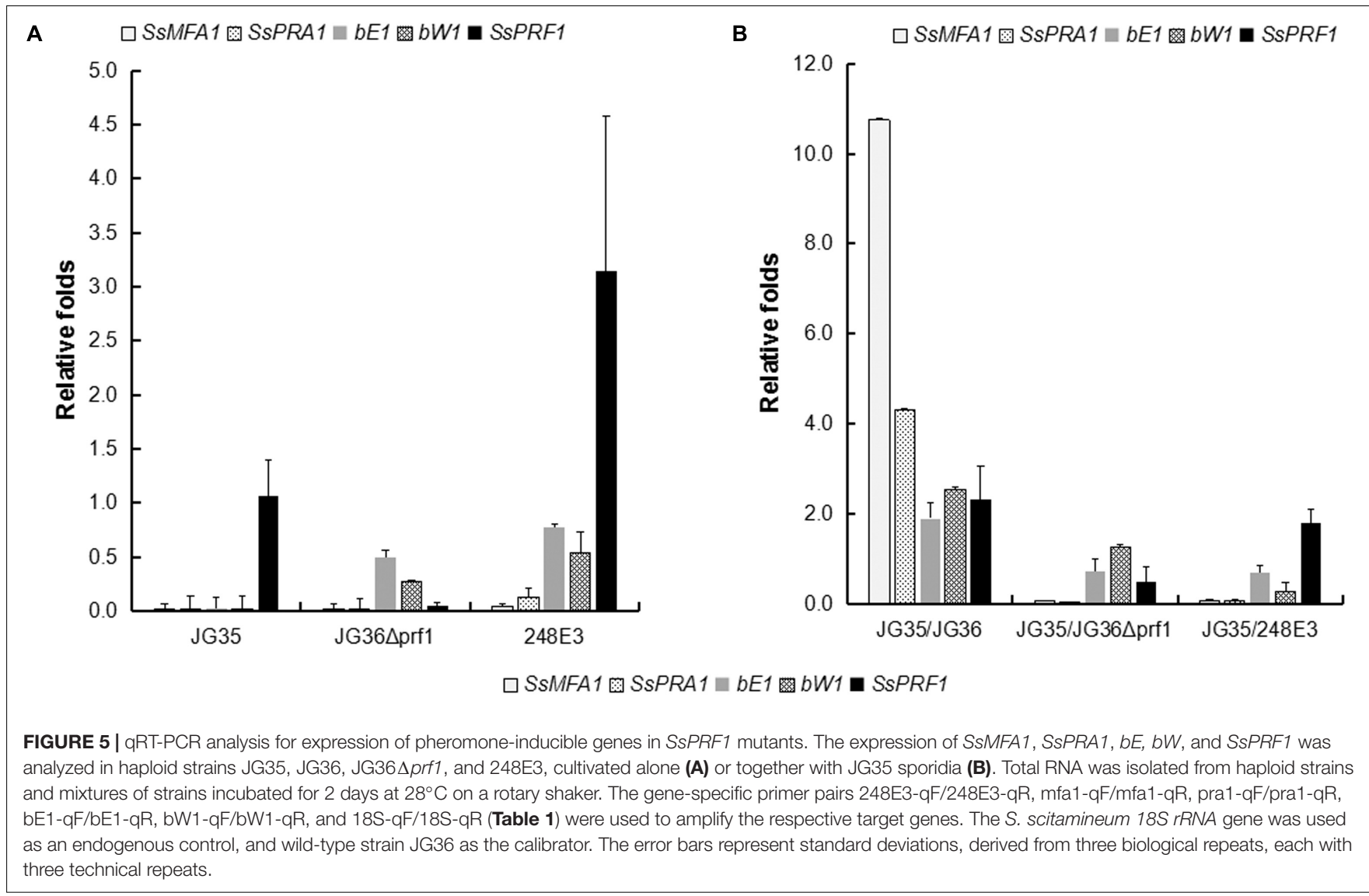

\section{DISCUSSION}

The morphological switch from yeast-like growth to filamentous growth occurs during the life style switch from the saprophytic to the biotrophic stage, and is critical for virulence of several animaland plant-pathogenic fungi (Hartmann et al., 1996; Urban et al., 1996; Nadal et al., 2008; Elías-Villalobos et al., 2015). The human fungal pathogen Candida albicans can switch from a unicellular yeast form into pseudohyphae or hyphae, and such transition is important for virulence (Lo et al., 1997; Wartenberg et al., 2014). In the fungal plant pathogen $U$. maydis, morphological switching from yeast-like sporidia to dikaryotic hyphae occurs after sexual mating between two cells of opposite mating-types, a process under regulation of biallelic $a$ and multiallelic $b$ loci (Bölker et al., 1992; Gillissen et al., 1992; Spellig et al., 1994). Sporisorium reilianum, a smut fungus closely related to U. maydis, possesses three $a$ alleles containing two active pheromone genes each, and at least five alleles for the $b$ locus, that govern its ability of sexual mating and dimorphic switching essential for virulence (Schirawski et al., 2005). S. scitamineum is also a dimorphic pathogen with two different life styles, a saprophytic stage growing by budding as unicellular sporidia, and a pathogenic stage growing as dikaryotic hyphae. The morphological switch in S. scitamineum also depends on sexual mating (Que et al., 2014), but its regulatory mechanism is still not fully understood. The conserved $b$ locus genes have also been functionally investigated, with the gene sequence encoding the pheromone receptors, $P R A 1$ and PRA2, annotated (Yan et al., 2016a,b). The MFA1 and MFA2 gene, respectively encoding the pheromone precursors in MAT1 and MAT-2 mating-type strain, have both been annotated and characterized by reverse genetics (Lu et al., 2017; Sun et al., 2019). However, the pheromone response factor Prf1 that governs pheromone-induced transcription of $a$ and $b$ loci in $U$. maydis, has not yet been identified or characterized in $S$. scitamineum. Neither was it characterized in the biological function of the pheromone receptor encoding genes, SsPRA1 and SsPRA2. As reported in this study, we identified a Prf1 ortholog in S. scitamineum and found that it was responsible for both basal and pheromone-induced expression of mating type genes of the a1 locus. We further showed that deletion of the a locus gene SsMFA1 or SsPRA1 resulted in similar phenotype (Figure 6A) as the T-DNA insertion mutation or deletion mutant of the SsPRF1 gene (Figure 3A), as well as the reported MFA1 deletion phenotype (Sun et al., 2019). Our results provide new insight into the mechanism of pathogenicity of this important sugarcane pathogen, by confirming the biological function of the al locus gene in S. scitamineum mating/filamentation, and indicating that SsPrf1 plays a key role in pheromone signaling and filamentous growth in S. scitamineum through transcriptional induction of the mating locus.

Under pheromone-induced conditions, expression of SsPRF1 in JG35/JG36 culture was about 2.4-fold up-regulated compared 


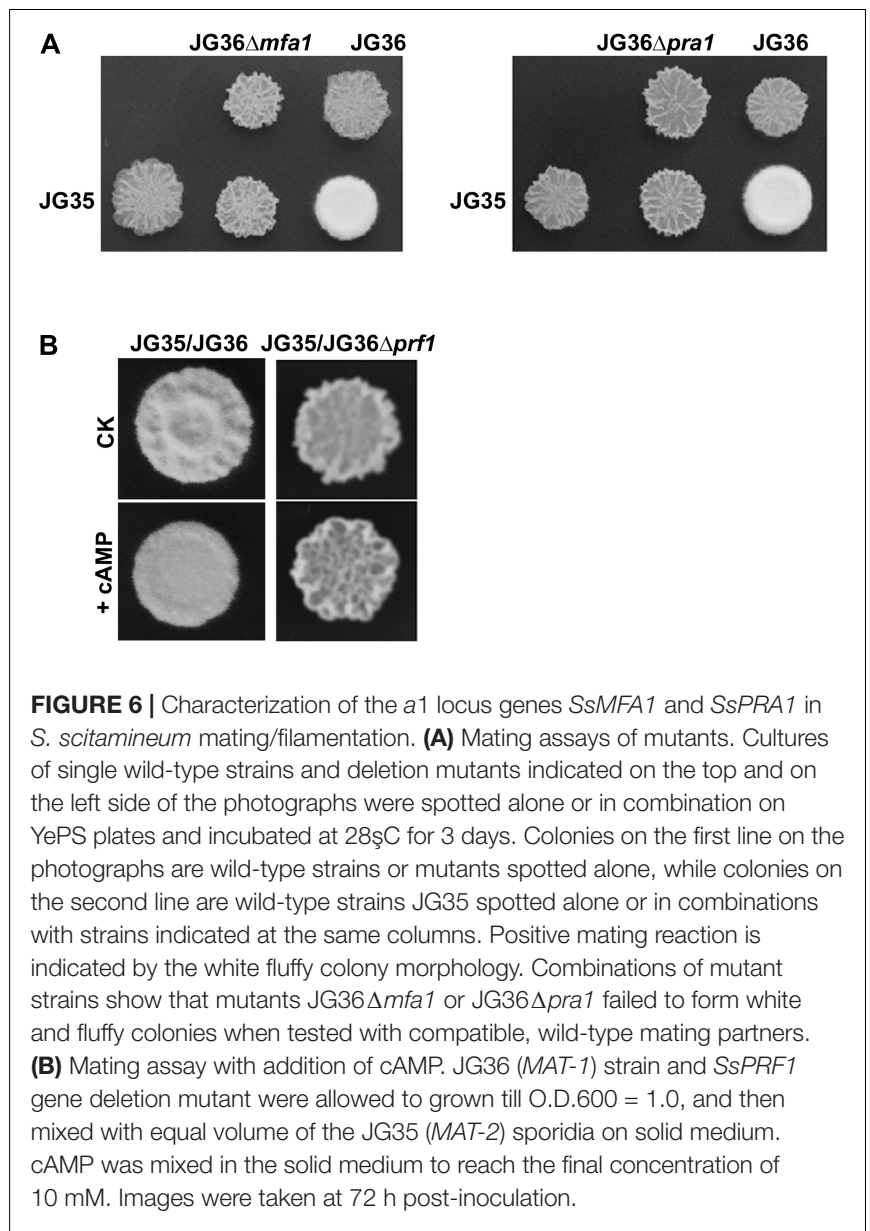

to that in the sporidial growth stage of the wild-type strains (Figure 5), suggesting that $S s P R F 1$ expression is up-regulated by mating. In this regard, it has been reported that in $U$. maydis Prf1 expression was significantly induced by sexual pheromones (Hartmann et al., 1996). Since SsPRF1 is present in both JG35 and JG36, we could not tell which strain may contribute to the increase in expression of the gene. By taking the expression levels in JG35/JG36 $\operatorname{pprf1}$ and JG35/284E3 into account, we concluded that truncated SsPRF1 in $284 \mathrm{E} 3$ was not functional, since the transcript level of SsPRF1 in JG35/284E3 was at about the average of JG35 and 284E3 (Figure 5). Studies in U. maydis have shown that Prf1 undergoes post-translational modification (phosphorylation) for activation (Kaffarnik et al., 2003). Thus it would be of interest to investigate the upstream signal transduction pathways that switch on pheromone-stimulated gene expression through SsPrf1 phosphorylation.

Chang et al. (2018) reported that cAMP-PKA signal pathway regulates $S$. scitamineum mating/filamentation likely through regulation of intracellular redox homeostasis. We found that exougenous addition of cAMP could not restore mating/filamentation of the $\Delta p r f 1$ mutant, as it does to the cAMP-PKA mutants (Chang et al., 2018), indicating that SsPrf1 acts at downstream of cAMP-PKA signaling pathway. This is consistent with what has been reported in $U$. maydis
(Kaffarnik et al., 2003). We found no obvious difference between the wild-type and the $\Delta p r f 1$ mutant in aspects of oxidative stress tolerance or intracellular $\mathrm{H}_{2} \mathrm{O}_{2}$ level (Supplementary Figures S4A,B). Also, addition of low concentration (0.1 mM) of $\mathrm{H}_{2} \mathrm{O}_{2}$ could not promote mating/filamentation in the $\Delta p r f 1$ mutant (under mating condition with the opposite mating-type sporidia, Supplementary Figure S4C), as it does to the wild-type strain or the cAMP-PKA mutants (Chang et al., 2018). These results confirmed that the SsPrf1 function is not relevant to intracellular redox homeostasis but may be solely on regulation of mating locus genes.

Overall, our present study, together with previous published functional study of $a$ and $b$ locus, completes the regulation network of $S$. scitamineum mating/filamentation at downstream of the cAMP-PKA signaling pathway, and in parallel of redox signaling.

\section{DATA AVAILABILITY}

All datasets generated for this study are included in the manuscript and/or the Supplementary Files.

\section{AUTHOR CONTRIBUTIONS}

GZ, YD, EC, MY, GC, ZW, CZ, and BZ performed the experiments. $\mathrm{PX}, \mathrm{BC}, \mathrm{CC}$, and $\mathrm{ZJ}$ conceived and designed the experiments. GZ, YD, CC, BC, and ZJ analyzed the data. GZ, $\mathrm{BC}$, and ZJ contributed reagents, materials, and analysis tools. GZ and YD wrote the manuscript. All authors read and approved the final manuscript.

\section{FUNDING}

This work was supported in part by the National Basic Research Program of China (973 Program, Grant No. 2015CB150600), National Natural Science Foundation of China (Grant No. 31660500), and Guangxi Key Laboratory of Biology for Crop Diseases and Insect Pests Project (Grant No. 15-140-45-ST1). The funders had no role in the design of the study, collection, analysis, and interpretation of data, and in the writing of the manuscript.

\section{ACKNOWLEDGMENTS}

We thank Dr. L. H. Ji and Dr. L. H. Sun for sharing the vectors pEX1-GAP-eGFP, pNGR1, and pEX2.

\section{SUPPLEMENTARY MATERIAL}

The Supplementary Material for this article can be found online at: https://www.frontiersin.org/articles/10.3389/fmicb.2019. 02115/full\#supplementary-material 


\section{REFERENCES}

Albert, H. H., and Schenck, S. (1996). PCR amplification from a homolog of the bE mating-type gene as a sensitive assay for the presence of Ustilago scitaminea DNA. Plant Dis. 80, 1189-1192.

Andrews, D. L., Egan, J. D., Mayorga, M. E., and Gold, S. E. (2000). The Ustilago maydis ubc4 and ubc5 genes encode members of a MAP kinase cascade required for filamentous growth. Mol. Plant Microbe Interact. 13, 781-786. doi: 10.1094/ mpmi.2000.13.7.781

Bakkeren, G., Kämper, J., and Schirawski, J. (2008). Sex in smut fungi: structure, function and evolution of mating-type complexes. Fungal Genet. Biol. 45(Suppl. 1), S15-S21. doi: 10.1016/j.fgb.2008.04.005

Bölker, M., Urban, M., and Kahmann, R. (1992). The a mating type locus of U. maydis specifies cell signaling components. Cell 68, 441-450. doi: 10.1016/ 0092-8674(92)90182-c

Brefort, T., Müller, P., and Kahmann, R. (2005). The high-mobility-group domain transcription factor Rop1 is a direct regulator of prf1 in Ustilago maydis. Eukaryot. Cell 4, 379-391. doi: 10.1128/ec.4.2.379-391.2005

Chacko, N., and Gold, S. (2012). Deletion of the Ustilago maydis ortholog of the Aspergillus sporulation regulator medA affects mating and virulence through pheromone response. Fungal Genet. Biol. 49, 426-432. doi: 10.1016/j.fgb.2012. 04.002

Chang, C., Cai, E., Deng, Y. Z., Mei, D., Qiu, S., Chen, B., et al. (2018). cAMP/PKA signalling pathway regulates redox homeostasis essential for Sporisorium scitamineum mating/filamentation and virulence. Environ. Microbiol. 21, 959971. doi: 10.1111/1462-2920.14496

Croft, B. J., and Braithwaite, K. S. (2006). Management of an incursion of sugarcane smut in Australia. Australas. Plant Pathol. 35, 113-122. doi: 10.1094/PDIS-1213-1257-RE

Dutheil, J. Y., Mannhaupt, G., Schweizer, G., Sieber, M. K. C., Münsterkotter, M., Güldener, U., et al. (2016). A tale of genome compartmentalization: the evolution of virulence clusters in smut fungi. Genome Biol. Evol. 8, 681-704 doi: $10.1093 / g b e / e v w 026$

Elías-Villalobos, A., Fernandez-Alvarez, A., Moreno-Sanchez, I., Helmlinger, D., and Ibeas, J. I. (2015). The Hos2 histone deacetylase controls Ustilago maydis virulence through direct regulation of mating-type genes. PLoS Pathog 11:e1005134. doi: 10.1371/journal.ppat.1005134

Felsenstein, J. (1985). Confidence limits on phylogenies: an approach using the bootstrap. Evolution 39, 783-791. doi: 10.1111/j.1558-5646.1985.tb00420.x

Gillissen, B., Bergemann, J., Sandmann, C., Schroeer, B., Bölker, M., and Kahmann, R. (1992). A two-component regulatory system for self/non-self recognition in Ustilago maydis. Cell 68, 647-657. doi: 10.1016/0092-8674(92)90141-X

Hajdukiewicz, P., Svab, Z., and Maliga, P. (1994). The small, versatile pPZP family of Agrobacterium binary vectors for plant transformation. Plant Mol. Biol. 25, 989-994. doi: 10.1007/bf00014672

Hartmann, H. A., Kahmann, R., and Bölker, M. (1996). The pheromone response factor coordinates filamentous growth and pathogenicity in Ustilago maydis. EMBO J. 15, 1632-1641. doi: 10.1002/j.1460-2075.1996.tb00508.x

Ji, L., Jiang, Z. D., Liu, Y., Koh, C. M., and Zhang, L. H. (2010). A Simplified and efficient method for transformation and gene tagging of Ustilago maydis using frozen cells. Fungal Genet. Biol. 47, 279-287. doi: 10.1016/j.fgb.2010.01.002

Jones, D. T., Taylor, W. R., and Thornton, J. M. (1992). The rapid generation of mutation data matrices from protein sequences. Comput. Appl. Biosci. 8, 275-282. doi: 10.1093/bioinformatics/8.3.275

Kaffarnik, F., Müller, P., Leibundgut, M., Kahmann, R., and Feldbrügge, M. (2003). PKA and MAPK phosphorylation of Prf1 allows promoter discrimination in Ustilago maydis. EMBO J. 22, 5817-5826. doi: 10.1093/emboj/cdg554

Kellner, R., Vollmeister, E., Feldbrügge, M., and Begerow, D. (2011). Interspecific sex in grass smuts and the genetic diversity of their pheromone-receptor system. PLoS Genet. 7:e1002436. doi: 10.1371/journal.pgen.1002436

Krüger, J., Loubradou, G., Regenfelder, E., Hartmann, A., and Kahmann, R. (1998). Crosstalk between cAMP and pheromone signalling pathways in Ustilago maydis. Mol. Gen. Genet. 260, 193-198. doi: 10.1007/s004380050885

Kumar, S., Stecher, G., and Tamura, K. (2016). MEGA7: molecular Evolutionary genetics analysis version 7.0 for bigger datasets. Mol. Biol. Evol. 33, 1870-1874. doi: 10.1093/molbev/msw054

Li, P., Gong, X., Jia, H., Fan, Y., Zhang, Y., Cao, Z., et al. (2016). MAP kinase gene STK1 is required for hyphal, conidial, and appressorial development, toxin biosynthesis, pathogenicity, and hypertonic stress response in the plant pathogenic fungus Setosphaeria turcica. J. Integr. Agric. 15, 2786-2794. doi: 10.1016/s2095-3119(16)61472-7

Liu, Y. G., and Chen, Y. (2007). High-efficiency thermal asymmetric interlaced PCR for amplification of unknown flanking sequences. Biotechniques 43, 649-650.

Livak, K. J., and Schmittgen, T. D. (2001). Analysis of relative gene expression data using real-time quantitative PCR and the 2(-Delta Delta C(T)) method. Methods 25, 402-408. doi: 10.1006/meth.2001.1262

Lo, H. J., Kohler, J. R., DiDomenico, B., Loebenberg, D., Cacciapuoti, A., and Fink, G. R. (1997). Nonfilamentous C. albicans mutants are avirulent. Cell 90, 939-949. doi: 10.1016/s0092-8674(00)80358-x

Lu, S., Shen, X., and Chen, B. (2017). Development of an efficient vector system for gene knock-out and near in-cis gene complementation in the sugarcane smut fungus. Sci. Rep. 7:3113. doi: 10.1038/s41598-017-03233-7

Marroquin-Guzman, M., and Wilson, R. A. (2015). GATA-dependent glutaminolysis drives appressorium formation in Magnaporthe oryzae by suppressing TOR inhibition of cAMP/PKA signaling. PLoS Pathog. 11:e1004851. doi: 10.1371/journal.ppat.1004851

Mayorga, M. E., and Gold, S. E. (1999). A MAP kinase encoded by the ubc3 gene of Ustilago maydis is required for filamentous growth and full virulence. Mol. Microbiol. 34, 485-497. doi: 10.1046/j.1365-2958.1999.01610.x

Mendoza-Mendoza, A., Eskova, A., Weise, C., Czajkowski, R., and Kahmann, R. (2009). Hap2 regulates the pheromone response transcription factor prf1 in Ustilago maydis. Mol. Microbiol. 72, 683-698. doi: 10.1111/j.1365-2958.2009. 06676.x

Müller, P., Aichinger, C., Feldbrügge, M., and Kahmann, R. (1999). The MAP kinase kpp2 regulates mating and pathogenic development in Ustilago maydis. Mol. Microbiol. 34, 1007-1017. doi: 10.1046/j.1365-2958.1999.01661.x

Müller, P., Weinzierl, G., Brachmann, A., Feldbrügge, M., and Kahmann, R. (2003). Mating and pathogenic development of the Smut fungus Ustilago maydis are regulated by one mitogen-activated protein kinase cascade. Eukaryot. Cell 2, 1187-1199. doi: 10.1128/ec.2.6.1187-1199.2003

Nadal, M., Garcia-Pedrajas, M. D., and Gold, S. E. (2008). Dimorphism in fungal plant pathogens. FEMS Microbiol. Lett. 284, 127-134. doi: 10.1111/j.1574-6968. 2008.01173.x

Que, Y., Xu, L., Wu, Q., Liu, Y., Ling, H., Liu, Y., et al. (2014). Genome sequencing of Sporisorium scitamineum provides insights into the pathogenic mechanisms of sugarcane smut. BMC Genomics 15:996. doi: 10.1186/1471-2164-15-996

Regenfelder, E., Spellig, T., Hartmann, A., Lauenstein, S., Bölker, M., and Kahmann, R. (1997). G proteins in Ustilago maydis: transmission of multiple signals? EMBO J. 16, 1934-1942. doi: 10.1093/emboj/16.8.1934

Saitou, N., and Nei, M. (1987). The neighbor-joining method: a new method for reconstructing phylogenetic trees. Mol. Biol. Evol. 4, 406-425.

Schirawski, J., Heinze, B., Wagenknecht, M., and Kahmann, R. (2005). Mating type loci of Sporisorium reilianum: novel pattern with three a and multiple b specificities. Eukaryot. Cell 4, 1317-1327. doi: 10.1128/ec.4.8.1317-1327.2005

Spellig, T., Bölker, M., Lottspeich, F., Frank, R. W., and Kahmann, R. (1994). Pheromones trigger filamentous growth in Ustilago maydis. EMBO J. 13, 1620 1627. doi: 10.1002/j.1460-2075.1994.tb06425.x

Sun, L., Yan, M., Ding, Z., Liu, Y., Du, M., Xi, P., et al. (2014). Improved dominant selection markers and co-culturing conditions for efficient Agrobacterium tumefaciens-mediated transformation of Ustilago scitaminea. Biotechnol. Lett. 36, 1309-1314. doi: 10.1007/s10529-014-1486-5

Sun, S., Deng, Y., Cai, E., Yan, M., Li, L., Chen, B., et al. (2019). The farnesyltransferase $\beta$-Subunit Raml regulates sporisorium scitamineum mating, pathogenicity and cell wall integrity. Front. Microbiol. 10:976. doi: 10.3389/fmicb.2019.00976

Sundar, A. R., Barnabas, E. L., Malathi, P., and Viswanathan, R. (2012). "A minireview on smut disease of sugarcane caused by Sporisorium scitamineum," in Botany, ed. J. Mworia, (Rijeka: InTech).

Taniguti, L. M., Schaker, P. D., Benevenuto, J., Peters, L. P., Carvalho, G., Palhares, A., et al. (2015). Complete Genome Sequence of Sporisorium scitamineum and biotrophic interaction transcriptome with sugarcane. PLoS One 10:e0129318. doi: 10.1371/journal.pone.0129318

Urban, M., Kahmann, R., and Bölker, M. (1996). Identification of the pheromone response element in Ustilago maydis. Mol. Gen. Genet. 251,31-37. doi: 10.1007/ bf02174341 
Wartenberg, A., Linde, J., Martin, R., Schreiner, M., Horn, F., Jacobsen, I. D., et al. (2014). Microevolution of Candida albicans in macrophages restores filamentation in a nonfilamentous mutant. PLoS Genet 10:e1004824. doi: 10. 1371/journal.pgen.1004824

Yan, M., Dai, W., Cai, E., Deng, Y. Z., Chang, C., Jiang, Z., et al. (2016a). Transcriptome analysis of Sporisorium scitamineum reveals critical environmental signals for fungal sexual mating and filamentous growth. $B M C$ Genomics 17:354. doi: 10.1186/s12864-016-2691-5

Yan, M., Zhu, G., Lin, S., Xian, X., Chang, C., Xi, P., et al. (2016b). The matingtype locus b of the sugarcane smut Sporisorium scitamineum is essential for mating, filamentous growth and pathogenicity. Fungal Genet. Biol. 86, 1-8. doi: 10.1016/j.fgb.2015.11.005

Zarnack, K., Eichhorn, H., Kahmann, R., and Feldbrügge, M. (2008). Pheromoneregulated target genes respond differentially to MAPK phosphorylation of transcription factor Prf1. Mol. Microbiol. 69, 1041-1053. doi: 10.1111/j.13652958.2008.06345.x

Conflict of Interest Statement: The authors declare that the research was conducted in the absence of any commercial or financial relationships that could be construed as a potential conflict of interest.

Copyright (C) 2019 Zhu, Deng, Cai, Yan, Cui, Wang, Zou, Zhang, Xi, Chang, Chen and Jiang. This is an open-access article distributed under the terms of the Creative Commons Attribution License (CC BY). The use, distribution or reproduction in other forums is permitted, provided the original author(s) and the copyright owner(s) are credited and that the original publication in this journal is cited, in accordance with accepted academic practice. No use, distribution or reproduction is permitted which does not comply with these terms. 